\title{
How to Diagnose Orofacial Pain?
}

\author{
Dra Mirta D'ambra* \\ Ministry of Health Agentina, University of Buenos Aires, Europe
}

Submission: July 30, 2018; Published: August 03, 2018

*Corresponding author: Dra Mirta D’ambra, Public Health Specialist Ministry of Health Agentina, University of Buenos Aires, Chairman Wams in Argentina Headquarters in Nedherlands, Email: mirtadambra@hotmail.com

\section{Mini Review}

It is usually incorrectly attributed to rhinosinusitis. Some patients describe pain as sinus. In a series of 973 patients with suspected rhinosinusitis, only 1 in 10 confirmed paranasal sinus disease by endoscopy and computed tomography (CT). Therefore, the correct diagnosis can be lost or delayed and result in inadequate treatment and prolonged symptoms. Chronic orofacial pain, which lasts more than 12 weeks and could have already received treatment in primary care, leading to the reconsideration of the diagnosis before the persistence of symptoms. What are the common causes of orofacial pain? Although there is no solid evidence on the prevalence of different conditions, migraine and middle facial segment pain are among the most common causes of orofacial pain. However, they are often misdiagnosed as rhinosinusitis due to the accompanying nasal symptoms.

\section{What do You Need to Know?}

a) Orofacial pain is not a hallmark of rhinosinusitis and affects only $10 \%$ of patients with rhinosinusitis.

b) Consider alternative diagnoses such as migraine, pain in the middle facial segment and the group of headaches that may present with facial pain and nasal symptoms, such as rhinorrhea and nasal congestion.

c) Start a therapeutic trial for 4 weeks for the most probable diagnosis and ask the patient to return if the symptoms do not improve.

\section{Rhinosinusitis}

Orofacial pain is not a hallmark of rhinosinusitis Rhinosinusitis is an inflammation of the lining of the nasal cavity and sinuses, with or without formation of nasal polyps. Symptoms can be acute $(<12$ weeks) or chronic ( $>12$ weeks). It is estimated that it affects $2-10 \%$ of the population. A study based on the population of Canada ( $n=73,000$ patients) reported a prevalence of $3.4 \%$ in men and $5.7 \%$ in women. Orofacial pain is not a hallmark of rhinosinusitis. The European Position Paper on Rhinosinusitis and Nasal Polyps (EPOS) defines rhinosinusitis and nasal polyps by the presence of 4 symptoms, of which, the presence of nasal block, congestion, obstruction nasal discharge (anterior rhinorrhea or postnasal drip) They are essential to support the diagnosis. So it is unlikely that patients with orofacial pain without congestion or anterior or posterior nasal obstruction have rhinosinusitis. The data from cohort studies show that orofacial pain is a poor predictor of chronic rhinosinusitis and it is more likely that, if pain is used as a criterion to evaluate patients with rhinosinusitis, an erroneous diagnosis is made [1]. A prospective observational study of 108 patients with confirmed chronic rhinosinusitis found that less than one third of patients had orofacial pain. When there are nasal polyps, the ratio drops to 1 in 10 patients, which indicates that orofacial pain is even less common in patients with chronic rhinosinusitis who have nasal polyps.

The International Headache Society states that «rhinosinusitis is not validated as a cause of headache or facial pain, unless it has an acute relapse.» Patients with acute rhinosinusitis are more likely to have orofacial pain, caused by irritation of the sensory nerves, through inflammatory mediators, changes in pressure and a sinus without drainage, blocked [2]. The pain is intense and is usually unilateral.

It is associated with fever and nasal obstruction, and in the case of acute maxillary rhinosinusitis, there may be dental pain. Recurrent true acute rhinosinusitis is rare, and the etiology of pain is often vascular, as in migraines or cluster headaches. As some patients with chronic orofacial pain may complain of associated nasal symptoms, it can be difficult to assess whether the pain is related to sinusitis, especially if the patient points out the pain clearly, at the anatomical sites of the sinuses, such as the cheeks or the front. Good and bad predictors of syngenic facial pain. Good predictive value Poor predictive value Increase in pain intensity when the patient moves from sitting to supine, or during a flight, or skiing Increases intensity when leaning forward Reduction of smell Normal smell. Improvement with antibiotic or corticosteroid treatment No improvement with antibiotic or corticosteroid treatment. Purulent, malodorous rhinorrhea Severe facial pain affects the activities of daily life Worse with upper respiratory tract infections Sensitivity or swelling on the skin of the face Symptoms of rhinosinusitis [3]. 
a) Nasal blockage, congestion, nasal obstruction: essential for diagnosis.

b) Nasal discharge (rhinorrhea or anterior or retronasal drip): essential for diagnosis

c) Facial pain

\section{Migraine}

Often, migraine is misdiagnosed as sinus headache or rhinosinusitis. Chronic incapacitating headaches are more likely to be related to migraines than to rhinosinusitis. EPOS states: «most sinus headaches can be classified as migraines.» For this reason, in patients presenting in primary care with «sinus headache», the diagnosis of migraine should be explored before considering rhinosinusitis. A recent systematic review of studies of migraine ( $>6$ million participants) reported a prevalence of 1 in 10 . While patients with migraine commonly report hemicrania, a subgroup of patients may be located on the face but with pain. characteristic symptoms of migraine, such as nausea, vomiting, sensitivity to light and sound. In a study of 517 patients with migraine, almost $9 \%$ had pain in the head and lower half of the face; these patients were also more likely to have associated trigeminal autonomic symptoms, such as rhinorrhea and nasal blockage. Often, migraine is misdiagnosed as sinus headache or rhinosinusitis, due to certain associated symptoms such as nasal congestion, rhinorrhea, lacrimation or inflammation of the cheeks, which appear during migraine attacks. In a prospective cohort study of 2,991 patients with a history of self-medication or of the International Headache Society. Of these patients, more than $80 \%$ of those who reported pain or sinus pressure, $63 \%$ reported nasal congestion and $40 \%$, rhinorrhea. How to make a diagnosis? The characteristics and site of pain can help distinguish these conditions through history and semiology.

It is more likely that the intense pain that interferes with the activities of the patient's daily life is motivated by a migraine or other headaches, unlike temporomandibular pain, middle facial segment pain and tension headache, which do not interfere. In general, the associated nasal symptoms are compatible with rhinosinusitis, but the authors recommend taking into account the autonomic trigeminal symptoms that appear in migraines, and the possibility that there is a coinciding rhinitis, given its high prevalence in the adult population. The history or family history of migraine are important. During the examination, the skin covering the breasts should be felt. If there is tenderness about the breasts, the suspicion of pain of the middle facial segment should be greater. It is necessary to feel on the temporomandibular joint and the neighboring areas, to detect sensitivity, reaching the temple and the line of the jaw, the mastoid area and the cheek, since within these areas are the trigger points for temporomandibular disorders. While the temporomandibular joint is palpated, the patient is asked to open and close the mouth to feel the click and laxity of the jaw. The laxity reflects a greater joint mobility and the protrusion of the joint laterally during the opening of the mouth. The laxity and the click of the jaw are signs of temporomandibular joint abnormality. The anterior rhinoscopy can be performed with an otoscope, to investigate the presence of purulent mucus in the nasal cavities (which supports the diagnosis of rhinosinusitis) or enlarged lower turbinates (suggesting rhinitis). The authors recommend a complete examination of the cranial nerves in all patients with chronic orofacial pain, to exclude intracranial tumors that may present with orofacial pain. In general, no studies are required to confirm the diagnosis.

\section{How is it Treated?}

Often, and due to overlapping symptoms, $\geq 2$ diagnoses are possible. You can start with a trial treatment for 4 weeks for the most likely diagnosis. If there is no improvement, said treatment is suspended and other diagnoses and alternative treatments are considered. If the patient has doubts about the diagnosis, it is important to explain the complex nature of orofacial pain, which can create diagnostic difficulties, because patients can find it reassuring to know that this situation is not rare. It is recommended to emphasize the need to follow up if the symptoms do not improve. The aforementioned symptoms nasal blockage, congestion, nasal obstruction, nasal discharge (rhinorrhea or anterior or retronasal drip) are sufficient to support the diagnosis of rhinosinusitis and begin treatment in primary care. The management of chronic rhinosinusitis depends on the severity of the symptoms and the presence of polyps. The EPOS guidelines recommend treatment with a steroid nasal spray and nasal spray, and then retest the patient at 4 weeks. If the symptoms have improved, then the treatment can be continued in the long term.

If it is a question of migraine or tension headache, advise patients to follow a daily routine, sleep hygiene, exercises, diet and periods of relaxation and, that reduce the consumption of caffeine. Patients with an acute episode of tension headache can be treated with $1 \mathrm{~g}$ of paracetamol or $400 \mathrm{mg}$ of ibuprofen. If the probable diagnosis is pain of the middle facial segment, low doses of amitriptyline $(10 \mathrm{mg}$ at night for at least 6 weeks) are recommended. This should be continued for a minimum of 6 months; If the condition has disappeared, treatment can be suspended as of this moment. The treatment of temporomandibular disorder is multifaceted, and includes lifestyle changes, relaxation techniques, simple analgesia with paracetamol or NSAIDs, low doses of benzodiazepines and neuropathic agents. Specialized treatment involves the use of temporomandibular joint injections, arthroscopies, arthroplasties, and joint replacement surgery.

\section{When is the patient referred?}

Cluster headache is the only condition that guarantees immediate referral to the neurologist. In most cases, only the referral of the patient is required when the symptoms are refractory to medical treatment initiated in primary care or, 
if there are associated alarm symptoms. Cluster headache is the only condition that guarantees immediate referral to the neurologist, due to the potential need for neuroimaging and specialized treatment. If the diagnosis is in doubt, or there are warning signs.

\section{References}

1. Macfarlane TV, Blinkhorn AS, Davies RM, Kincey J, Worthington HV (2002) Oro-facial pain in the community: prevalence and associated impact. Community Dent Oral Epidemiol 30(1): 52-60.
2. Conti PCR, Pertes RA, Heir GM, Nasri C, Cohen HV, et al. (2003) Orofacial pain: basic mechanisms and implication for successful management. J Appl Oral Sci 11: 1-7.

3. West B, Jones NS (2001) Endoscopy negative, computed tomography negative facial pain in a nasal clinic. Laryngoscope 111: 581-586.

\section{Your next submission with Juniper Publishers will reach you the below assets}

- Quality Editorial service

- Swift Peer Review

- Reprints availability

- E-prints Service

- Manuscript Podcast for convenient understanding

- Global attainment for your research

- Manuscript accessibility in different formats ( Pdf, E-pub, Full Text, Audio)

- Unceasing customer service

Track the below URL for one-step submission https://juniperpublishers.com/online-submission.php 